\title{
Agrobacterium-mediated Transformation of Squash (Cucurbita pepo L.) to induce Virus Resistance by Gene Silencing
}

\author{
Y. Khidr, M. Wallbraun, T. Wetzel, G. Krczal and G. M. Reustle \\ RLP AgroScience GmbH, AIPlanta - Institute for Plant Research, Breitenweg 71, D-67435 Neustadt/W, Germany \\ Correspondence to: Y. Khidr, RLP AgroScience GmbH, AlPlanta - Institute for Plant Research, Breitenweg 71, D-67435 Neustadt/W, Germany
}

Received: September 5, 2005

Transformation of plants with an inverted repeat constructs containing plant virus derived cDNA, separated by an intron as spacer have been shown to produce double stranded RNA (dsR$\mathrm{NA}$ ) that can results in a virus resistance by post-transcriptional gene silencing.

In this study, highly conserved regions of the coat protein genes of Watermelon mosaic virus-2 (WMV-2) and Zucchini yellow mosaic virus (ZYMV) was converted into cDNA by RTPCR and constructed as inverted repeats. These constructs were cloned into a binary vector under the control of the $35 \mathrm{~S}$ promoter.

As target tissue for genetic transformation somatic embryogenesis was induced from different organs (leaves, shoot tips, cotyledons and matured seeds) of three squash cultivars. The explants were incubated on MS medium supplemented with different combination of auxin and cytokinin. Embryogenic callus developed within 13-18 weeks of incubation on all different explants of the three squash cultivars. Efficiency of embryo maturation, germination and conversion into entire plant from squash embryogenic callus was found to be callus age depended.

Agrobacterium-mediated transformation of squash embryogenic callus was established by transient GUS expression. Effect of four Agrobacterium strains, inoculum concentration, acetosyringone concentration during infection and/or cocultivation and period between subculture and infection were examined. Transformation efficiency was best with the supervirulent ATHV strain, at optical density of $0.85,1 \mathrm{mM}$ acetosyringone in the infection medium, and when embryogenic callus was used 5-9 days after subculture on fresh medium.

Experiments to transform embryogenic callus of squash with the inverted repeat constructs containing WMV-2 and ZYMV sequences are on the way. 